\title{
Author Index Vol. 30, 1994
}

Achiron, A. 157 Aguglia, E. 85 Ansseau, M. 1 Appelberg, B. 20 Appelboom, Th. 66 Arolt, V. 114 Avanzini, P. 15

Balogh, S.E. 197 Beneke, M. 37 Benkert, O. 101 Benton, D. 106 Bertolino, A. 85 Beyer,JJ. 178 Birch, NJ. 73 Blair, R.D.G. 189 Blois, R. 79 Bock, A. 178 Bode, M. 114 Bosmans, E. 57 Brambüla, F. 15

Caccavari, R. 15 Caekebeke, J.F.V. 143 Carlen, P.L. 189 Casacchia, M. 85 Christodulopulu, E. 178 Ciani, N. 85 Cocito, L. 148 Costelli, P. 29 Delsignore, R. 15 Devlin, J.G. 153 Diefenbacher, A. 94 Dijk, J.G.van 143 Dubey, G.P. 185

Eggert, F. 42

Ferstl, R. 42 Fertonani-Affini, G. 15 Fitzpatrick, D.F. 197 Fontanesi, B. 15 Fonzi, S. 29 Fransolet, L. 66

Friedrich, A. 94 Fritze, J. 37 Fukushima, T. 7, 46 Furuta, H. 7

Gaebel, W. 94 Gaillard, J.-M. 79 Galimberti, CA. 29 Gerra, G. 15 Giberti, L. 148 Glover, BJ. 132 Guo, X. 132 Gupta, B.S. 185 Gupta, U. 185

Hambalek, R. 4 Hansenne, M. 1 Hayakawa, H. 53 Hendricks, S.E. 197 Herning, R.I. 132 Hisada, K. 46 Holsboer, F. 101 Hurme, M. 20

Invernizzi, G. 85

James, J.E. 124 Jibiki, I. 7,46

Kagara, Y. 7 Katila, H. 20 Kempenaers, Ch. 66 Kido, H. 46 Killeen, AA. 24 Kinoshita, T. 126 Kirov,|G.K. 73 Kishizawa, S. 7 Kline, J.P. 197 Kömpf, D. 114

Kretz, M. 178 Krishnan, B. 4 Kurokawa, K. 7,46

Lal, S. 4 Laor, N. 157 
Lehmann, D. 126 Lehmann, W. 11 Lehnert, H. 178 Leonard, B.E. 24

Mackert, A. 94 Maertelaer, V. de 66 Maes, M. 57 Maestri,D. 15 Maj, M. 61 Manni, R. 29 Marcato, A. 15 Marchesi, G.F. 85 Martino, M. 61 Matsuda, H. 46 Meltzer, H.Y. 57 Mendlewicz, J. 66 Merica, H. 79 Michel, CM. 126 Mingard, P. 66 Mirel, J. 1

Möller, H.-J. 94,160 Monteleone, P. 61 Moreno, A.G. 1 Motohashi, N. 53 Müller, H. 94 Müller, MJ. 173 MüUer-Ruchholtz, W. 42 Murialdo, G. 29 Nardini, M. 85 NgYingKin, N.M.K 4 Nishida, A. 53 Noldy, N.E. 189 Noy, S. 157

O'Keeffe, ST. 153 Owens, D.S. 106

Paige, S.R. 197 Parodi, C, 29 Perna, G. 15 Piccolo, A. 61 Pitchot, W. 1 Politzer, N. 189 
Polleri, A. 29 Primavera, A. 148

Ramsey, R.G. 73 Rao, M.L. 160 Rapisarda, V. 85 Rasmus, W. 37 Rimón, R. 20 Rød, I.-S. 37 Rybakowski, J.K 11

Saito, M. 126 Santos, C.V. 189 Schulz, C. 178 Scotto, P. 148 Shimizu, M. 53 Signoret, P. 79 Simenon, G. 66 Solinas, G.P. 29 Song, C. 24 Steadman, P. 73 Steiger, A. 101 Stock, G. 94 Sweden, B. van 143

Tartara, A. 29 Thavundayil, J.X. 4 Torre, F. 29 Torrecilas, J.G. 1

Uharek, L. 42

Vander Elst, M. 66 Verleger, R. 114 Volz, H.-P. 94

Wahlbeck, K. 20 Wascher, E. 114

Yagyu, T. 126 Yamaguchi, N. 7,46 Yamawaki, S. 53

202 